\title{
PERFORMANCE EVALUATION OF 3DPD, THE PHOTOGRAMMETRIC PIPELINE FOR THE CASSIS STEREO IMAGES
}

\author{
C. $\operatorname{Re}^{1 *}$, S. Tulyakov ${ }^{2}$, E. Simioni ${ }^{1}$, T. Mudric ${ }^{1}$, G. Cremonese ${ }^{1}$, N. Thomas ${ }^{3}$ \\ ${ }^{1}$ INAF Osservatorio Astronomico di Padova, Vicolo dell’Osservatorio, Padova, Italy (cristina.re@inaf.it) \\ ${ }^{2}$ Dept. of Ecole Polytechnique Federale de Lausanne (EPFL), Lausanne, Switzerland, \\ ${ }^{3}$ Physics Institute, Space Research and Planetary Sciences - University of Bern, Bern, Switzerland.
}

Commission III, ICWG III/II

KEY WORDS: Stereo, Photogrammetry, CaSSIS, DTM, Accuracy

\begin{abstract}
:
A novel photogrammetric pipeline has been designed by INAF-Padova for the processing of the recent stereo images of CaSSIS and it will be a starting point for the future procedures that will be applied to Stereo Camera (STC) (Cremonese, 2009; Da Deppo, 2010) images for the BepiColombo mission to Mercury. The large number of stereo pairs being generated has made it necessary that several teams attempt to generate products. The presented procedures are the two strategies (proposed by INAF-PADOVA and by EPFLLausanne) available nowadays in an international attempt to generate 3D products from the CaSSIS images. The comparisons here presented will be the first of several such efforts and are important to make the planetary community aware of the accuracy of the 3D data available. Furthermore, the possibility to consider higher accuracy DTMs as the ones of HiRISE makes the quality assessment of stereo products of CaSSIS robust and important for the assessment of data to be provided to the scientific community. The performance evaluation of the INAF-Padova pipeline (3DPD software) is the main objectives of this work. Additionally, the comparison between the correlation phase of 3DPD and of ASP (Moratto, 2010) that is integrated in the EPFL pipeline has been considered.
\end{abstract}

\section{INTRODUCTION}

The CaSSIS instrument (Colour and Stereo Surface Imaging System) (Thomas, 2017), is the stereo imaging system onboard the European Space Agency's ExoMars Trace Gas Orbiter (TGO) that has been launched on 14 March 2016 and entered Mars orbit on 19 October 2016.

TGO is placed in a $400 \mathrm{~km}$ circular orbit with an inclination of $74^{\circ}$ providing more opportunities to monitor mid-latitudes, but nominally none over polar surface regions $\left(> \pm 74^{\circ}\right.$ latitudes).

The top level scientific objectives of the imager on TGO are (1) to characterise sites which have been identified as potential sources of trace gases, (2) to investigate dynamic surface processes (e.g. sublimation, erosional processes, impacts, and potential volcanism) which may help to constrain the atmospheric gas inventory, and (3) to characterise potential future landing sites by measuring local (down to $\sim 10 \mathrm{~m}$ ) slopes. CaSSIS, the main imaging system on the spacecraft, is able to provide two set of images of the same target from two different points of view along the same orbit. After acquisition of the first set of images, the telescope is rotated of $180^{\circ}$ so the second set is then acquired. The stereo pairs can then be aligned to provide the $3 \mathrm{D}$ topography of specific targets. The system operates in "pushframe" mode and provides stereo pairs fundamental for the initialisation of the photogrammetric process to $3 \mathrm{D}$ reconstruct the Martian surface (Figure 1).

The camera combines stereo and multispectral capabilities enhancing the ability of the science team to investigate terrain and geology. It provides images at different wavelengths thanks to a FSA (Filter Strip Assembly) with a panchromatic (PAN, centred at $675 \mathrm{~nm}$ ) and 3 broadband colour filters within the visible range (the BLU filter centred at $499 \mathrm{~nm}$, the RED one at $836 \mathrm{~nm}$ and the NIR one centred at $937 \mathrm{~nm}$ ). All the stereo data products generated by the different institutes and science teams involved in the CaSSIS project will be archived in a repository set up at INAF (National Institute of Astrophysics) of Padova by the team of the institute.
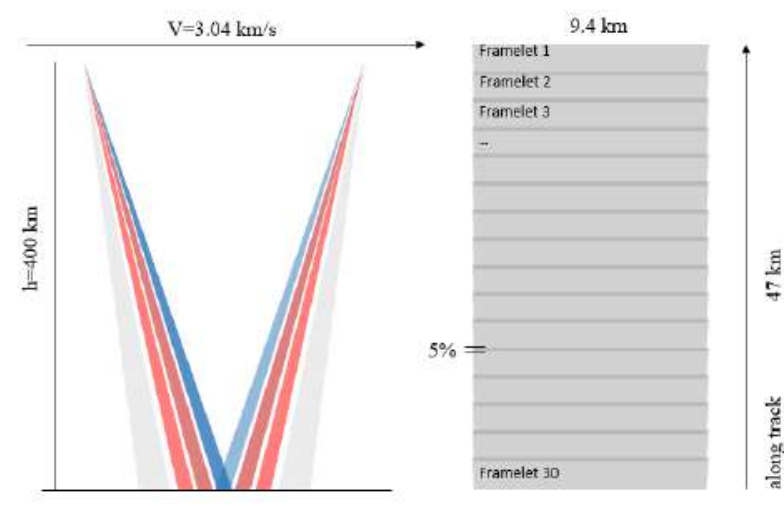

Figure 1. CaSSIS observation strategies. On the left: PAN, RED, NIR, BLU filters FoV for the first acquisition and after the rotation of the mechanism. On the right: the on-ground footprints.

The Repository is accessed through a website (http://cassis.oapd.inaf.it/), it provides the ability of on-line visualization and download of the stereo products that are delivered periodically after their validation.

Among the activities concerning the Repository configurations, software and tools for DTM generation from CaSSIS images have been developed by the INAF team. 3DPD (3 Dimensional reconstruction of Planetary Data) (Simioni et al. 2017) is an 
efficient pipeline that starting from the stereo image preprocessing, through a photogrammetric process, is able to provide accurate three-dimensional data.

In parallel, the team at EPFL adopted publicly available NASA Ames Stereo Pipeline for processing CaSSIS stereo images. To interface the pipeline with the CaSSIS they developed custom mosaicking and triangulation procedures. The mosaicking procedure performs bundle adjustment, map-projection and stitching of the stereo sequences.

In the paper two types of tests have been performed. In the first, the matching accuracy of 3DPD has been checked against that of ASP and with respect to a reference DTM produced by the University of Arizona with Socet Set (SS) with HiRISE (HighResolution Imaging Science Experiment) (McEwen, et al. 2007) stereo images as also tested in (Re et al. 2012).

Since the comparison of the image matching procedure is the objective of the first test, all other steps of the DTM generation procedure have been made independent of the matching software by using the same framework for mosaicking and triangulation developed by EPFL (the code and Ubuntu executable are available online (Tulyakov, 2019)).

In the second test, the performance evaluation of the INAFPadova pipeline has been evaluated considering the HiRISE DTMs are data reference.

\section{SOFTWARE USED FOR DTM GENERATION}

\subsection{DPD}

The complete workflow, starting from the CaSSIS framelets up to the final DTM generation is described in Figure 2. The pipeline contains some sequential steps: the creation of the map-projected mosaics from the framelets, the definition of an initial disparity map, the disparity refinement at the sub-pixel level and the triangulation phase arriving to the DTM production.

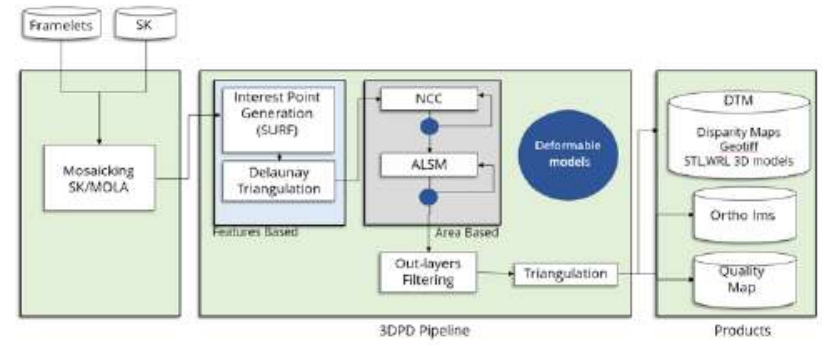

Figure 2. 3DPD workflow

\subsubsection{The Matching Core}

In order to improve the correlation performances we decided to start the process using an interest operator (SURF) (Bay et al, 2008) which allows to identify a number of conjugated points. Once defined a set of the consistent points using a RANSAC (RANdom SAmple Consensus) estimation, the disparity for each point is computed. Then, extracting a starting disparity map applying a Delaunay triangulation, the correlation initialization with an NCC (Lewis, 1995), at the first level of the pyramid, is executed. Consecutively, for each level of the image pyramid, a sub-pixel refinement by area based matching (Least-Squares Matching) is execute (Gruen, 1985).

The matched points are then transferred to the next level (of higher resolution) where the disparity of the additional points is predicted from the neighborhoods with a bilinear interpolation technique, providing the starting location (approximate values) for the sub-pixel refinement by area based matching (LeastSquares Matching) in the search image.

\subsection{Ames Stereo Pipeline}

The mosaics resulting from the EPFL pipeline are fed to ASP that performs stereo matching. The image matching program ASP is an open source suite of fully automated geodesy and stereogrammetry tools designed to process planetary imagery captured from robotic explorers (Broxton et al, 2008; Moratto et al, 2010) from the NASA Ames Intelligent Robotics Group. The program is integrated with the updated versions of USGS ISIS software package (Torson and Becker, 1997).

The ASP matching core consists in finding for every point in one mosaic its physically corresponding one in an another mosaic. The stereo matching is performing in vertical and horizontal directions using patch-based cross-correlation with sub-pixel refinement (default settings of ASP).

\section{STEREO DATASETS}

\subsection{CaSSIS stereo pairs}

The stereo datasets used for the investigations analysed in this paper consists of three CaSSIS stereo pairs.

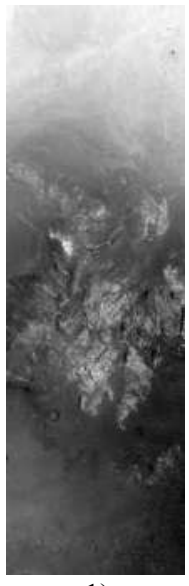

1)

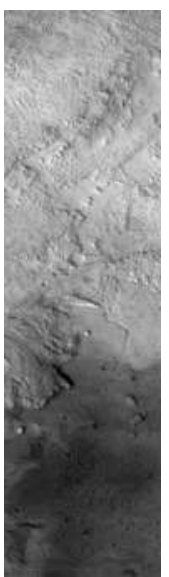

2)

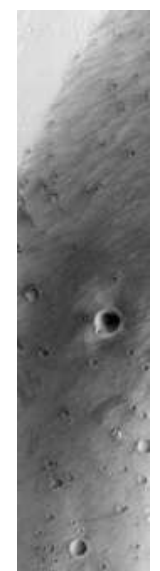

3)
Figure 3. The first images of the three stereo pairs considered in the analysis.

Here a description of the three different datasets:

1. The observation frame a central structure of a large impact crater. The CaSSIS stereo pair (MY34_004219_201_1/2) have been acquired the 2018-11-03 at 09:33:06.553. The region is located at $-18.6569^{\circ}$ Latitude and $62.6211^{\circ}$ Longitude and it has been covered by the PAN-NIR-BLU+PAN-RED-BLU colour filter combination mode.

2. The second observation is on the Jezero crater exactly where ancient rivers flowed into the crater. The CaSSIS stereo pair (MY34_004069_162_1/2) have been acquired in date 2018-1116 at $2 \overline{1}: 39: 57 . \overline{779}$. The region is located at $-23.9146^{\circ}$ Latitude 
and $326.4139^{\circ}$ Longitude and it has been covered by the PANRED+PAN-NIR-BLU colour filter combination mode.

3. The third observation is on the candidate landing site for 2020 ExoMars Mission in Gusev Crater. The CaSSIS stereo pair (MY34_004041_196_1/2) have been acquired in date 2018-1019 at 19:54:54.878. The region is located at $-14.657^{\circ}$ Latitude and $175.6412^{\circ}$ Longitude and it has been covered by the PANNIR+PAN-BLU colour filter combination mode.

\subsubsection{DPD mosaicking strategy}

The expected final pipeline products are images that are calibrated both radiometrically and geometrically comprising framelets stitched together to make complete swaths over the target. The length of the swath depends upon the number of framelets requested and the exact altitude. The number of framelets is constrained by the memory available in the Digital Processing Module (DPM) and the time needed to execute the rotation. The swath lengths in the range $40-50 \mathrm{~km}$ are typical.

The mosaicking strategy developed by INAF group follows several steps.

The SPICE Kernels provide both the interior camera parameters and the external orientation parameters describing the instantaneous position and attitude of the telescope and of each framelet. After the distortion removal, a fast strategy that exploits the stereo geometry of the acquisitions has been implemented to obtain the best stereo rectified image pair to be processed. Images have been rectified projecting them on a plane at a height identified by the intersection of the boresight direction with the MOLA surface.

The images are then resampled through a bilinear interpolation. In the overlapping regions where the parallax error is minimized, the intensity values correspond to a weighted mean between the overlapping framelets. The weight factor changes according to a r-cosine function

\subsubsection{EPFL-Lausanne mosaicking strategy and triangulation}

The mosaicking process is performed using python scripts based on USGS ISIS, which are available online (Tulyakov, 2019). At first, framelets of each sequence are converted to USGS ISIS cube format, de-noised (only BLU and NIR) and bundle-adjusted using "jigsaw" function. The de-noising consists of $3 \times 3$ median filtering followed by $3 \times 3$ boxcar filtering. The bundle adjustment is performed using framelets from all available channels. During the bundle adjustment, the camera rotation angles are adjusted for each frame to minimize the frame projection error subject to constraint that the adjustments should not be too large. Next, the common projection map is determined based on ground overlap of the sequences from the stereo observation. Using the common projection map, framelets of each sequence are independently map-projected and mosaicked. The mosaics include information allowing to trace back origin of every pixel in the mosaic to the original framelets. The resulting map-projected PAN mosaics are then used for the stereo matching.

As final step, the triangulation is performed using custom $\mathrm{C}++$ USGS ISIS application even available online (Tulyakov, 2019). For every matched pair of pixels from the mosaics the triangulation procedure finds corresponding framelets using trace information in the mosaics. Then it recovers camera vectors corresponding to these points using information from the framelets and computes the $3 \mathrm{D}$ point as an approximate intersection of these vectors. Each 3D point is then projected to the closest surface point of Mars ellipsoid. The elevation at map coordinates is then determined from these points using nearest neighbourhood interpolation. Note that the built-in triangulation of ASP can not be used since the mosaics do not preserve information about camera pointing and spacecraft position.

\section{QUANTITATIVE AND QUALITATIVE ASSESSMENTS}

In order to assess the quality of the DTMs determined by stereophotogrammetry, from a metrical point of view, the estimated vertical accuracy will be taken in account. Also a qualitative assessment of the abundance and appearance of artefacts in the DTM will be considered.

\subsection{Accuracy Evaluation}

The vertical accuracy can be estimated by collecting statistics on the differences between the stereo DTM and another topographic data of higher accuracy that can be used as reference ("ground truth") (Kirk et al. 2003). The topographic products provided by the laser altimeter (MOLA) (Smith et al, 2001) can be considered as reliable reference data since the laser measurement have normally a higher height accuracy and a high degree of geometric consistency on a regional and global scale. The comparisons are useful especially where the resolutions are similar between the two datasets.

CaSSIS stereo pair covering a region acquired by a higher or comparable resolution camera (such as CTX and HiRISE) can be used to evaluate the vertical accuracy of DTMs. In this investigation the HiRISE DTMs have been used as reference.

\subsubsection{Reference DTMs}

All the HiRISE DTM used as reference in the comparisons have been produced by the University of Arizona and retrieved from the Hirise website (http://hirise.lpl.arizona.edu). The SOCET SET (SS) software from Bae System applying the NGATE (Ref) strategy has been used to extract terrains.

The first study case refers to the stereo acquisition (ESP_021494_1610, ESP_013134_1610). The DTM (DTEED_0214̄94_1610_013134_1610_A01.IMG) has been generated with a post spacing of $2 \mathrm{~m}$. The total RMS computed from the discrepancies with respect to MOLA and reported by the producer is $4.6099 \mathrm{~m}$.

The second reference DTM (DTEEC_002387_1985_003798_1985_A01.IMG) comes from the HiRISE source images (PSP_002387_1985, PSP_003798_1985) and it has been produced with a post spacing of $1 \mathrm{~m}$ and with a total RMS of $4.733 \mathrm{~m}$.

The third reference DTM (DTEEC_036087_1655_035164_1655_U01.IMG) comes from the HiRISE source images (ESP_036087_1655, ESP_035164_1655) and it has been produced with a post spacing of 1 m and with a unknown total RMS reported. 


\subsection{Comparisons between DTMs}

The aim of the investigation is to evaluate the quality of the CaSSIS DTMs produced respectively with the 3DPD stereo pipeline and with the EPFL-Lausanne pipeline. The estimation of the vertical accuracy of the $3 \mathrm{D}$ product come from the extraction of the statistics (RMS) of the discrepancies between the CaSSIS DTM and the HiRISE DTM used as reference because of higher vertical precision. The comparison process has been performed by a 3D Modelling software able to manage the DTMs converted in TIN format or point clouds. Though this system all the systematic inconsistencies are removed easily and a best-fit automatic alignment can be performed through an ICP (iterative closest point) algorithm already implemented.

Due to the different orientation procedure between SS, ASP and 3DPD, therefore, geo-referencing inconsistencies arise. The procedure started with a point-to-surface alignment to register the two datasets then an iterative alignment process by ICP has been run to finally analyze only the differences in shape.

All the DTM involved in the comparison were previously converted to a TIN (Triangulated Irregular Network) representation, then, the signed distance of each triangulated surface from the reference one is computed. Due to the preliminary point-to-surface alignment, the average error is almost zero and therefore the Root Mean Square Error (RMSE) is close to the Std. Dev. of the discrepancies.

The peculiarity of the CaSSIS acquisition process make the influence of interior and exterior orientation parameters critical for the final restitution accuracy. Infact, the persisting misalignments between the framelets after the mosaicking stage affect even the final triangulation process for the DTM extraction.

\subsubsection{Comparisons of the matching cores}

Since our interest, at this stage, is focused on comparing just the level of performance of the matching algorithms (3DPD and ASP strategies), we opted to compute object coordinates using the same mosaicking and triangulation codes (framework of Tulyakov), so that the differences that occur in the comparisons depend only on the matching phase.

In particular, the mosaicking and the triangulation routines implemented in the standalone package of Tulyakov were used. In this way, only the two stereo matching phases (disparity maps) during the comparison of both the $3 \mathrm{D}$ results evaluated with respect the HiRISE reference DTM.

Summarising, the procedure implemented in the standalone package has provided the mosaicked images for both the two strategies considered, then, in one case, the disparity maps provided by the ASP matching core is given as input in the triangulation process; on the other side, the 3DPD disparity maps derived by the same mosaics have initialized the triangulation process of the standalone.

\section{Case 1: Central crater structure}

The accuracy of the DTM reconstruction is measured as RMSE of the discrepancies between the DTMs reconstructed, in the first case, by the 3DPD matching core applying LSM approach and, in the second case, by the ASP that implements the sub pixel refinement (mode 2), both respect to the HiRISE reference DTM.

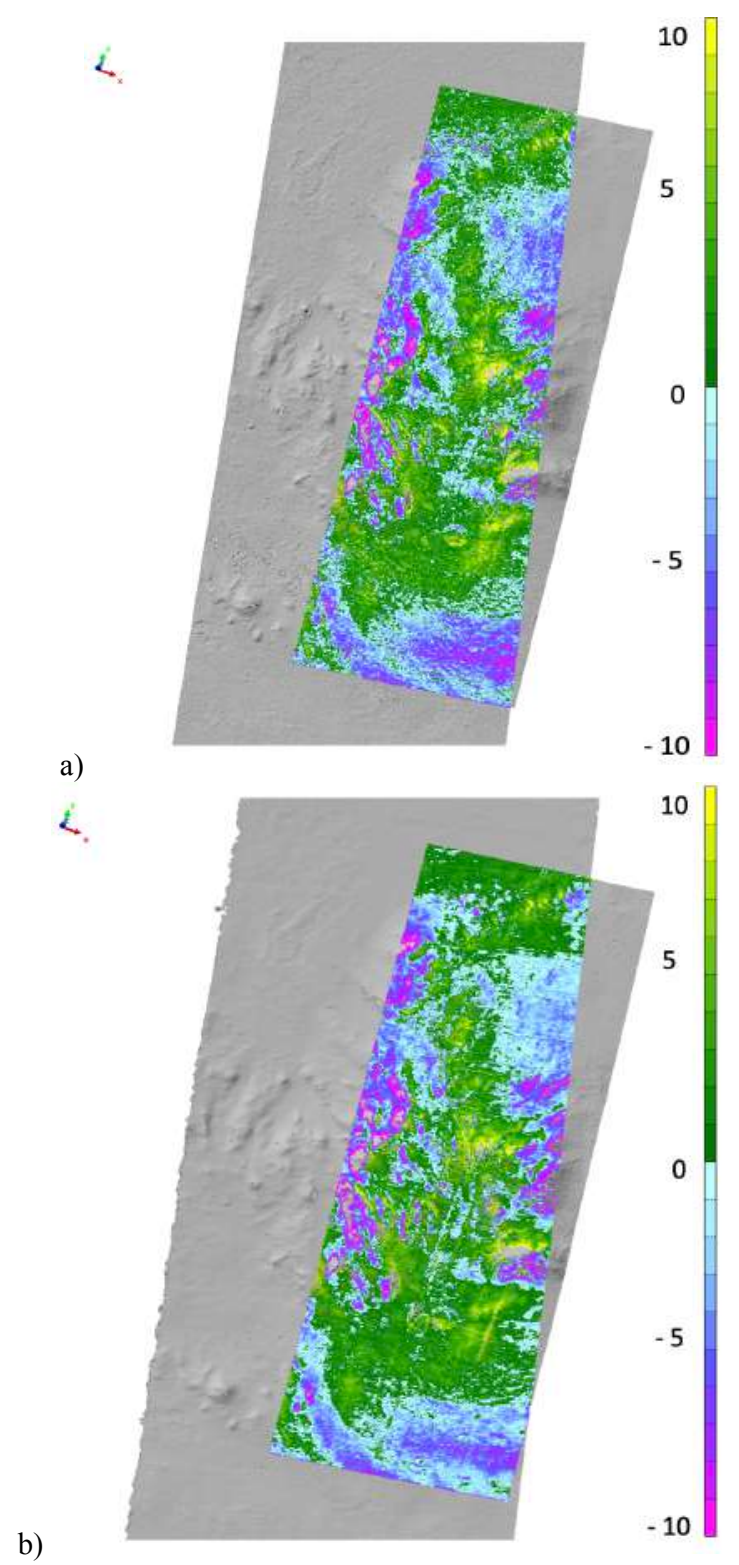

Figure 3: a) Colour map of the discrepancies extracted for the comparison between 3DPD DTM and HiRISE DTM.

b) Colour map of the discrepancies extracted for the comparison between ASP DTM and HiRISE DTM.

Figure 3 shows the RMSE both in graphical forms (color coded maps) as well as numeric forms Table 1-2 (for the first study case).

Table 1: 3DPD statistics

\begin{tabular}{|l|l|}
\hline \#Points & 463043 \\
\hline Mean [m] & -0.016 \\
\hline StdDev [m] & 3.78 \\
\hline RMS Error [m] & 3.78 \\
\hline
\end{tabular}

Table 2: EPFL statistics

\begin{tabular}{|l|l|}
\hline \#Points & 427386 \\
\hline Mean $[\mathrm{m}]$ & -0.018 \\
\hline StdDev [m] & 3.54 \\
\hline RMS Error [m] & 3.54 \\
\hline
\end{tabular}


From the results, we can notice that the matching cores compared provide DTMs with very close results in terms of RMSE values, The second pipeline provide a bit higher values of accuracy but the number of points considered in the statistical analysis is a bit lower probably because a number of points were not considered in the statistic since they are rejected by the threshold imposed in the comparison. The threshold has been fixed to $10 \mathrm{~m}$ equal to almost 2 pixels on ground. Also from the graphical analysis in Figure 4 reporting the profiles extracted in a small region (Case 1) from both the DTMs, the discrepancies are quite small and both the DTMs fit well the shape of the terrain.

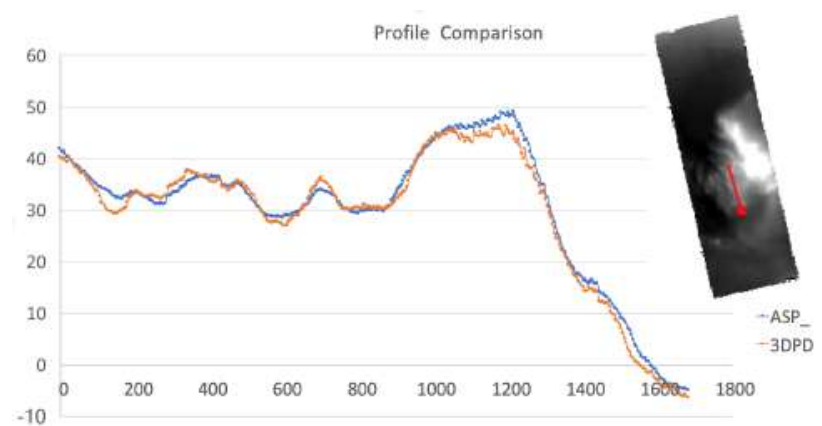

Figure 4: Profiles extracted from the section (red line) on the DTM (on the right side) produce by the implementation of the ASP matching core (Blue line) and by the 3DPD matching core (Orange line).

\subsubsection{Evaluation of the INAF-PADOVA pipeline}

In the second Test, the overall stereo strategy (proposed INAFPADOVA) has been evaluated.

The DTMs produced has been compared using HiRISE as reference and the discrepancies statistics have been collected. Is important to underline that the strategy adopted by 3DPD does not apply any Bundle Block adjustment procedure during the mosaicking of the images (stitching of the framelets). Even though, an overall Bundle adjustment procedure has been applied taking advantage of some ground control points (GCPs) on the MOLA data. The manual extraction of some recognizable features on the 3D map have been used to improve the internal consistency of our DTMs and re-estimate the projection matrices associated to the approximated single center of projection associated at the global mosaics and used during the triangulation process.

The final results in terms of RMS can be affected by the missing correction of the pointing parameter of each single framelets as in the Case 2 where the RMSE is the higher and the number of the points considered in the comparison is lower.

In the Tables below, the RMS Errors of the 3D products are reported.

\section{Case 1: Central crater structure}

Table 3: INAF-PADOVA statistics

\begin{tabular}{|l|l|}
\hline \#Points & 311394 \\
\hline Mean $[\mathrm{m}]$ & -0.009 \\
\hline StdDev [m] & 5.25 \\
\hline RMS Error [m] & 5.25 \\
\hline
\end{tabular}

Case 2: Jasero Crater

Table 4: 3DPD statistics

\begin{tabular}{|l|l|}
\hline \#Points & 232981 \\
\hline Mean $[\mathrm{m}]$ & 0.0004 \\
\hline StdDev [m] & 5.31 \\
\hline RMS Error [m] & 5.31 \\
\hline
\end{tabular}

Case 3: Gusev Crater

Table 5: 3DPD statistics

\begin{tabular}{|l|l|}
\hline \#Points & 273351 \\
\hline Mean [m] & 0.0042 \\
\hline StdDev [m] & 4.60 \\
\hline RMS Error [m] & 4.60 \\
\hline
\end{tabular}

\subsection{Qualitative DTM Analysis}

For each DTM generated by the 3DPD pipeline, a Quality Map $(\mathrm{QM})$ has been produced. These maps show the stereo correlation quality for each pixel (elevation post) in the DTM. The correlation quality is defined by a threshold imposed on the NCC (Normalized Cross Correlation) (Lewis, 1995) coefficient. The quality categories are identified in the legend as reported in the next Table 3 .

The values of the QM are automatically collected by 3DPD as part of the stereo correlation for the output DTM. The QM shows the color-coded categories draped over a shaded relief map as in Figure 5.

Table 3: Quality map Legend

$$
\begin{array}{lc}
\text { Low } & N C C_{A f f}<0.5 \\
\text { Fidelity } & \\
\text { Medium } & 0.5<N C C_{A f f}<0.7 \\
\text { Fidelity } & N C C_{A f f}>0.7 \\
\text { High } & \\
\text { Fidelity } & \\
\text { Interp2 } & \text { Snakes Based }
\end{array}
$$
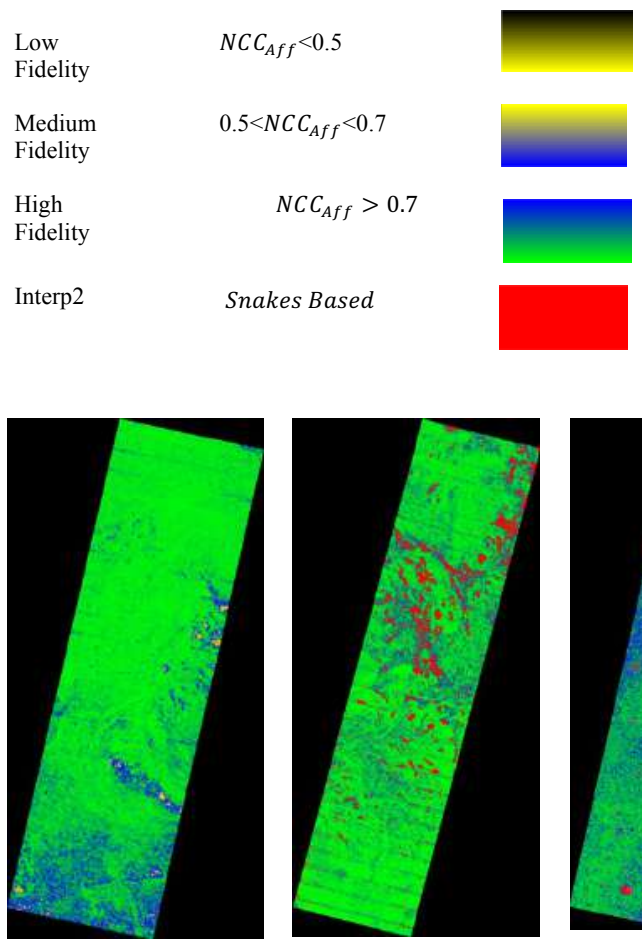

a)
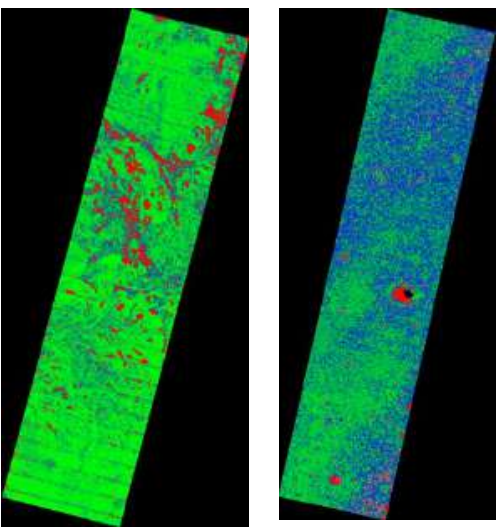

b)

c)

Figure 5: Quality maps of the three case studies.

The Case 1 has the best overall quality with respect to the others, the percentage of high fidelity points is higher as clearly visible from Figure 4 with majority of green-blue areas. The details and 
features present on the surface have been better resolved with respect to the other two study cases.

The inspection of the shaded relief version of the DTMs is also useful for qualitative assessment of resolved details, noise characteristics, height artefacts and surface patterns. The Figure 5 shows the comparison between the two strategies (Case 1). In the 3DPD case is visible a better extraction of some details of the surface with the second strategy more flattened and less able to resolve small features of the surface.
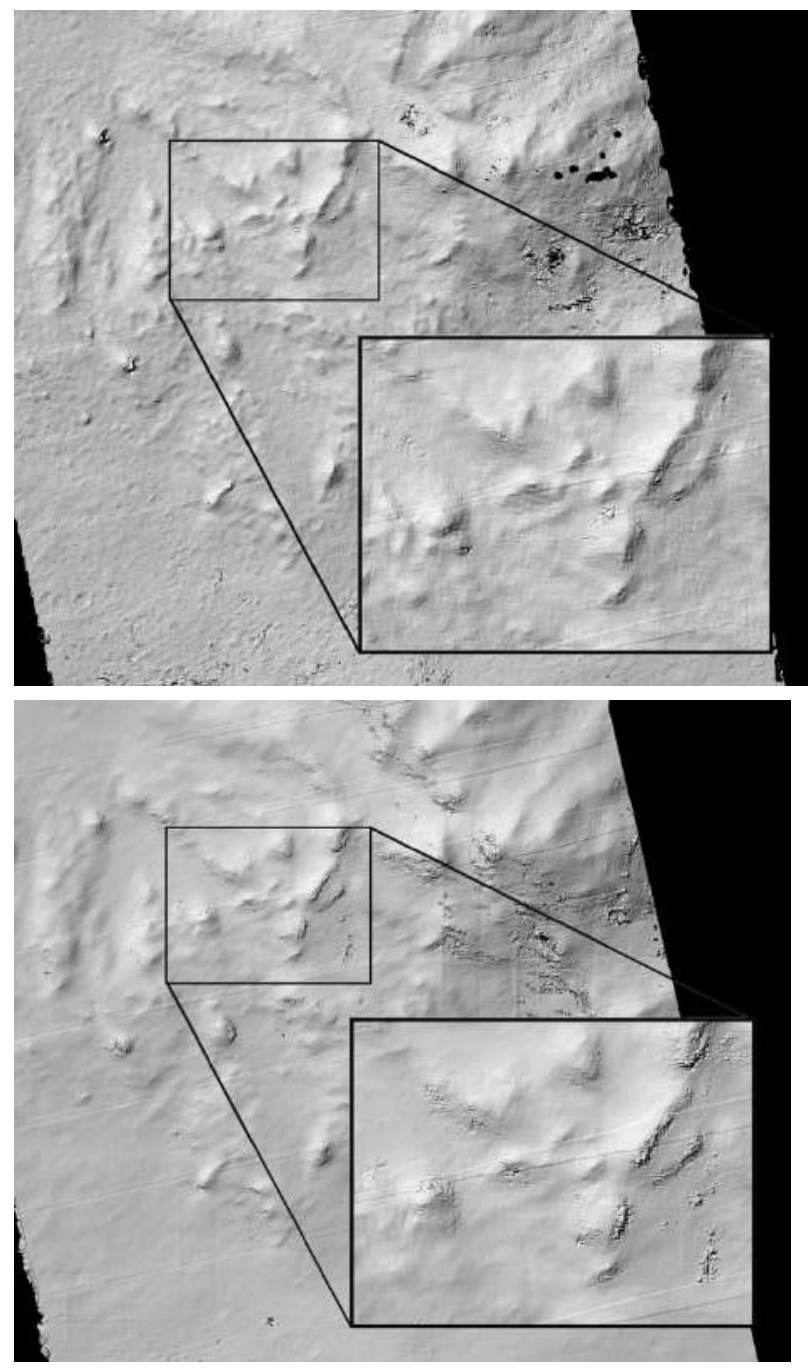

Figure 5: Top: Shaded relief version of the DTM (Case 1) generated with 3DPD strategy, Bottom: Shaded relief version of the DTM (Case 1) generated with Losanne-ST strategy

Furthermore, the colour coded overlay (Figure 6) can be used for the visualization of gross artefacts and internal model deformation or lateral shifts but not are suited for fine details.

With the colour coded visualisation, the possibility to recognise the impact of the framelets misalignment is significant. Especially in the case 3, the misalignments are quite evident.
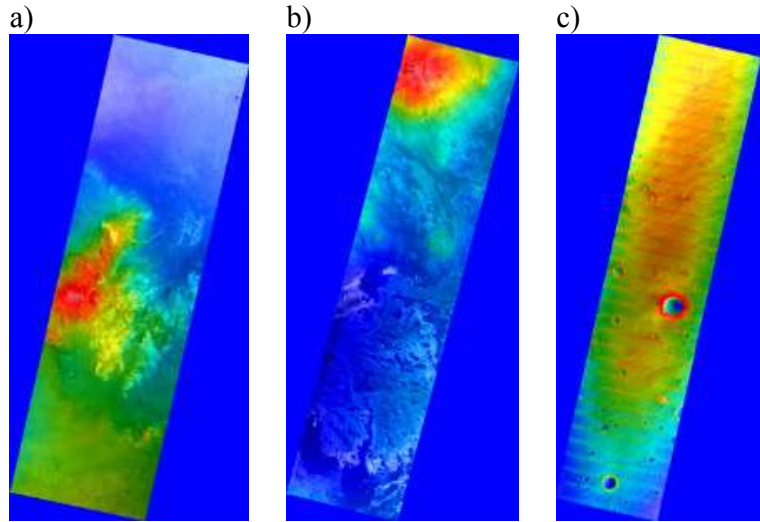

Figure 6: Colour coded overlay for the three study cases (case $1,2,3$ )

\section{CONCLUSIONS}

In conclusion, this work gives an overview of the actual state of art of the DTM generation using CaSSIS images. The comparisons, against the HiRISE DTMs, used to evaluate the DTMs quality, were useful to provide a quantitative assessment of the 3D products also provided to scientific the community through the public repository at INAF-Padova.

The pipelines that are available in the international panorama provide good and comparable results in terms of accuracy.

The three study cases provide accuracy values in the order of magnitude of around one pixel on ground (nominally $4.7 \mathrm{~m}$ at $400 \mathrm{~km})$.

The application of a Bundle Adjustment between framelets is mandatory to obtain better results and the removal of the gaps through this iterative procedure will be the next attempt for the group of Padova.

\section{ACKNOWLEDGEMENTS}

The authors would like to express their gratitude to the CaSSIS team for the calibration during the commissioning phase and the Italian Space Agency (ASI) for providing us the opportunity to give our contribution to the project (ASI-INAF agreement no.I/018/12/0).

\section{REFERENCES}

Bay, H. et al., 2008. Speeded-up robust features(SURF). Computer vision and image understanding 110.3: pp.346-359. CVIU, Vol. 110, No. 3, pp. 346-359.

Broxton, M. J., Edwards, L. J. 2008. The Ames Stereo Pipeline: Automated 3D Surface Reconstruction from Orbital Imagery. Lunar and Planetary Science Conference 39, abstract \#2419.

Cremonese, Gabriele, et al. "The stereo camera on theBepiColombo ESA/JAXA mission: a novel approach." Advancesin Geosciences 15 (2009): 305.

Da Deppo, V., et al. "Preliminary results of the opticalcalibration for the Stereo Camera STC onboard the BepiColombomission." International Conference on Space Optics. Vol. 7.2014. 
Gruen, Armin. Adaptive least squares correlation: a powerfulimage matching technique. South African Journal ofPhotogrammetry, Remote Sensing and Cartography 14.3 (1985):175-187.

Lewis, John P. "Fast normalized cross-correlation." Visioninterface. Vol. 10. No. 1. 1995.

Malin, Michael C., et al. "Context camera investigation onboard the Mars Reconnaissance Orbiter." Journal of GeophysicalResearch: Planets 112.E5 (2007).

McEwen, Alfred S., et al. "Mars reconnaissance orbiter'shigh resolution imaging science experiment (HiRISE)." Journalof Geophysical Research: Planets 112.E5 (2007).

Miller, S. B., and A. S. Walker, Die Entwicklung der digitalenphotogrammetrischen Systeme von Leica und Helava, Z.Photogramm. Fernerkundung,1(95), 4-16, 1995.

Moratto, Z. M., et al. "Ames Stereo Pipeline, NASA's opensource automated stereogrammetry software." Lunar andPlanetary Science Conference. Vol. 41. 2010.

Re, C., Roncella, R., Forlani, G., Cremonese, G., Naletto,G., 2012. Evaluation of area based image matching applied toDTM generation with Hirise images. ISPRS Ann. Photogramm.Rem ote Sens. Spat. Inf. Sci. Vol I-4, 209-214.

Simioni, E., et al. "A Photogrammetric Pipeline for the 3D Reconstruction of CaSSIS images on board ExoMars TGO." The International Archives of Photogrammetry, Remote Sensing and Spatial Information Sciences 42 (2017): 133-139.

Smith, David E., et al. Mars Orbiter Laser Altimeter: Experiment summary after the first year of global mapping of Mars. Journal of Geophysical Research: Planets 106.E10(2001): 23689-23722.

Thomas, Nicolas, and Gabriele Cremonese. "The Colour and Stereo Surface Imaging System for ESA's Trace Gas Orbiter." EGU General Assembly Conference Abstracts. Vol. 18.2016.

Torson, J.M., and Becker, K.J., (1997) ISIS - A Software Architecture for Processing Planetary Images (abs.), LPSC XXVIII, pp. 1443-1444.

Tulyakov, Stepan, Anton Ivanov, Nicolas Thomas, Victoria Roloff, Antoine Pommerol, Gabriele Cremonese, Thomas Weigel, and Francois Fleuret. "Geometric calibration of colour and stereo surface imaging system of ESA's Trace Gas Orbiter." Advances in space research 61, no. 1 (2018): 487-496.

Tulyakov S., Running NASA Ames Pipeline with TGO CaSSIS, https://github.com/tlkvstepan/CaSSIS_stereo, Accessed: 01 April 2019.

Kirk, R. et al. (2003), High- resolution topomapping of candidate MER landing sites with Mars Orbiter Camera narrowangle images, J. Geophys. Res., 108(E12), 8088, doi:10.1029/2003JE002131. 